\title{
L'industrie microtechnique en Franche-Comté : un système productif complexe en cours de structuration territoriale
}

The microtechnical industry in Franche-Comté: a complex production system in the process of territorial restructuring

Mikrotechnische Industrie in der Region Franche-Comté : ein Produktionssystem im territorialen Strukturationsprozess

\section{Sophie Carel}

\section{OpenEdition}

\section{Journals}

Édition électronique

URL : http://journals.openedition.org/rge/1517

DOI : $10.4000 /$ rge. 1517

ISSN : 2108-6478

Éditeur

Association des géographes de l'Est

Édition imprimée

Date de publication : 1 juin 2007

ISSN : 0035-3213

\section{Référence électronique}

Sophie Carel, «L'industrie microtechnique en Franche-Comté : un système productif complexe en cours de structuration territoriale », Revue Géographique de l'Est [En ligne], vol. 47 / 3 | 2007, mis en ligne le 04 novembre 2011, consulté le 08 septembre 2020. URL : http://journals.openedition.org/rge/ 1517 ; DOI : https://doi.org/10.4000/rge.1517

Ce document a été généré automatiquement le 8 septembre 2020.

Tous droits réservés 


\title{
L'industrie microtechnique en Franche-Comté : un système productif complexe en cours de structuration territoriale
}

\author{
The microtechnical industry in Franche-Comté: a complex production system in \\ the process of territorial restructuring \\ Mikrotechnische Industrie in der Region Franche-Comté : ein Produktionssystem \\ im territorialen Strukturationsprozess
}

Sophie Carel

\section{Introduction}

1 Enjeu de la politique européenne et par conséquent des politiques nationales, l'innovation est au cœur des problématiques de développement économique, notamment dans les pays qui, comme la France, ont à faire des efforts dans ce domaine pour atteindre les objectifs de Lisbonne fixés à l'échéance 2010 en matière de recherche-développement ${ }^{1}$.

2 Ce nouveau cadre européen implique pour les États de formuler des politiques d'innovation pertinente. La tendance générale est celle de la constitution ou le développement de pôles caractérisés par une masse critique de recherchedéveloppement suffisante et une mise en réseau des acteurs et des ressources de l'innovation.

3 La France conforte ainsi son soutien aux réseaux d'entreprises localisés par le lancement, fin 2004 d'une nouvelle politique industrielle en faveur des pôles de compétitivité. Il s'agit d'augmenter les capacités d'innovation d'un secteur et de le positionner dans la compétition européenne voire mondiale en créant de grands pôles puissants rassemblant universités, entreprises et organismes de formation dans des 
projets coopératifs. Le CIADT du 12 juillet 2005 a repéré en France 15 pôles de dimension internationale ${ }^{2}$ et 52 pôles " à vocation nationale ou régionale " qui semblent avoir au mieux une visibilité nationale.

4 C'est dans cette dernière catégorie de pôles un peu anonymes que la région FrancheComté a vu classer son pôle de compétitivité microtechnique.

5 L'industrie microtechnique comtoise et son nouveau pôle de compétitivité se doivent de répondre aux nouveaux enjeux de structuration territoriale et de mise en réseau des acteurs de l'innovation. Mais elle soulève depuis quelques décennies de nombreux débats et questions, tant au niveau du monde académique qu'industriel ou politique. Malgré la complexité du système microtechnique, peut-on considérer les microtechniques comme une spécialisation de la région Franche-Comté ? Quelles sont les dynamiques territoriales de cette industrie et en particulier a-t-elle des bases locales?

\section{Les microtechniques en Franche-Comté : un système productif complexe}

6 Reconnue capitale de l'horlogerie pendant deux siècles, Besançon et sa région affichent leur spécialisation microtechnique depuis la fin des années 1970. L'ancrage local et la réalité économique semblent ne faire aucun doute pour les acteurs locaux, d'autant que la zone d'emploi de Besançon a été labellisée successivement par la DATAR système productif local (SPL) microtechnique ${ }^{3}$ puis la région labellisée pôle de compétitivité, suggérant ainsi un territoire microtechnique organisé, avec des collaborations fortes entre industrie, formation et recherche.

\section{A. Les microtechniques : une notion et un secteur ambigus}

7 Pourtant, il existe un contraste saisissant entre la promotion dont le secteur jouit au niveau régional et la difficulté que l'on a de représenter le potentiel microtechnique. D'un point de vue théorique, la définition des microtechniques et son périmètre ne font pas consensus, ni dans le monde académique, ni chez les industriels. Les microtechniques n'ont rien de formalisé ; elles se sont construites progressivement sur plus de quatre décennies, en réponse à la crise des activités horlogères. La notion même reste vague puisqu'elle désigne les techniques du petit et du précis. D'un point de vue industriel, les microtechniques renvoient à une multitude de technologies, de métiers, de secteurs d'activité et de PME qui ont peu de relations entre elles et qui sont disséminées sur tout le territoire. L'extrême transversalité sectorielle, la multitude de produits et les non moins nombreuses technologies induites par les microtechniques empêchent un recensement strict des activités et entreprises relevant du secteur.

8 Plus concrètement, rechercher ce qui peut faire l'unité des quelques 350 entreprises dites microtechniques (UIMM et Comité des microtechniques de Franche-Comté, 2005), réparties sur toute la région et travaillant pour des secteurs d'activité nombreux relève de l'impossibilité.

9 Le potentiel microtechnique est donc loin d'être évident à révéler et a fait l'objet de nombreuses recherches académiques ou non (Cheref, 1996 ; CESR, 2001 ; Maillat et al., 2002, 2003). Tous ces travaux se contentent d'approximations en repérant, à partir des 
matériaux existants (nomenclatures d'activités, annuaires, répertoires...) des activités ou des entreprises microtechniques. Finalement, on aboutit toujours soit à une sousestimation, soit à une sur-estimation du secteur.

\section{B. Le territoire microtechnique, une problématique encore à délimiter}

10 La question de l'organisation de l'industrie microtechnique et de la nature de ses activités reste donc en suspens. La question de son territoire est également difficile à élucider. En l'absence de toute possibilité d'analyse statistique, la démarche inductive reste la seule voie de recherche possible.

11 Dans cette optique, le croisement de trois types d'analyses (activités et provenance géographique des exposants du salon Micronora ${ }^{4}$, effectifs et localisation des formations microtechniques et des emplois) révèle que le système microtechnique semble se développer de manière préférentielle en Franche-Comté, Ile-de-France et Rhône-Alpes. Une première hypothèse est que les compétences locales peuvent être présentes sur d'autres territoires, mais sous un autre nom.

12 Mais on peut également envisager l'existence d'une nouvelle forme de système productif, pluri-territorial, qui fonctionnerait de manière réticulaire, avec des spécificités territoriales (Carel \& Larceneux, 2004 ; Carel, 2003). Il convient de se souvenir que l'organisation de la production horlogère dont est issue l'industrie microtechnique présentait un cas de figure similaire. Même si elle débouchait sur un produit unique (la montre), elle s'était développée avec des grandes spécialisations territoriales sur les types de composants. Les compétences n'étaient pas les mêmes entre la Vallée de l'Arve (décolletage principalement), le Haut-Doubs, la région de Besançon ou celle de Montbéliard (découpage et traitement de surface). Le district industriel horloger était situé en fait sur plusieurs territoires inter-reliés, mais étroitement spécialisés. Il est possible que les entreprises issues de l'horlogerie et reconverties dans les microtechniques se soient réorganisées à une autre échelle géographique, en s'intégrant à quelques zones industrielles spécifiques. Si les microtechniques se partagent effectivement entre trois territoires, la région FrancheComté doit se positionner de manière plus claire, surtout par rapport à Grenoble (pôle Minalogic), dont le secteur de la microélectronique est très structuré. La région, marginalisée du champ de la microélectronique, aurait certainement intérêt à se positionner sur des axes non concurrents de Grenoble (la mécanique, le micromécanisme de silicium...).

13 Malgré la complexité du système microtechnique, l'essentiel est de savoir si les microtechniques possèdent les caractéristiques propres d'un pôle de compétitivité, au sens d'une coopération entre acteurs de la formation, de la recherche et des entreprises.

\section{La structuration territoriale des microtechniques}

\section{A. De 1960 à 2001 : les difficultés de structuration territoriale}

14 La crise horlogère des années 1970-1980 a conduit acteurs horlogers et pouvoirs publics à s'interroger sur l'avenir industriel de la région et sur les moyens de fédérer les 
acteurs de l'ancien territoire horloger. Les technologies et les compétences horlogères traditionnelles (production d'objets de petite dimension) paraissaient complémentaires à celles de l'industrie électronique et ses exigences de miniaturisation. Tout au long des années 1980, acteurs locaux publics et privés, se sont ralliés sous la bannière des microtechniques, qui laissaient présager des débouchés nombreux, dans l'automobile, l'aéronautique, le médical...

Le système éducatif a joué un rôle moteur dans cette évolution : c'est lui qui, le premier, a été conscient de la nécessaire "modernisation " à insuffler à un système marqué par deux siècles de tradition mécanique. Le système éducatif a donc progressivement fait évoluer la filière professionnelle dans ce sens, mais trente années d'évolution n'ont pas suffi à assurer une meilleure adéquation avec les besoins des entreprises. Le système éducatif, résolument tourné vers les microtechniques, est confronté à une production locale qui relève essentiellement de la micromécanique. Dans de telles conditions, les relations entre industrie, recherche et éducation restent difficiles.

Le désir de maintien d'un système productif local, relève donc essentiellement d'un microcosme formé d'enseignants, de chercheurs, de techniciens... gravitant autour du système éducatif. Néanmoins, on ne peut que constater l'échec relatif de la diversification totale vers les microtechniques. Déjà, l'industrie comtoise est restée sur les composants mécaniques et doit donc recourir à l'approvisionnement externe de composants électroniques puisqu'elle n'a pas la maitrise de ce segment de production : ceci est un premier signe de recomposition du système sur des bases qui ne sont plus strictement locales. De plus, l'essaimage des microtechniques est limité à quelques cas exceptionnels comme la création de l'entreprise Statice par d'anciens ingénieurs de Lip $^{5}$. Ensuite, l'écart n'a cessé de se creuser entre le tissu industriel régional, basé essentiellement sur la micromécanique, et les institutions (centres de recherche, collectivités locales et appareil de formation) qui ont souhaité une orientation vers les microtechniques. Enfin, les entreprises, très en amont des filières, sont souvent en position de sous-traitance face à de grands donneurs d'ordre. Il est donc difficile de faire jouer des solidarités et de créer des institutions propres au secteur comme pour l'horlogerie...

17 Malgré les espoirs nourris autour des microtechniques - une reconversion a priori réalisable d'une industrie horlogère en crise - il restait difficile, encore tout récemment, d'identifier localement un système productif microtechnique.

La faiblesse des relations entre acteurs de l'éducation, de la recherche et de l'industrie est révélatrice des conditions mêmes de développement du territoire et de la nature des savoir-faire issus de la longue tradition horlogère.

$\mathrm{Au}$ total, tous ces constats ne plaident pas en faveur de l'existence d'un système productif localisé microtechnique. Premièrement, au regard de la proximité, les liens entre université et industrie sont faibles. Les entreprises sont trop petites pour avoir accès à la recherche, elles n'ont pour la plupart pas de service RechercheDéveloppement proprement dit, recherche et production occupant souvent les mêmes personnes. Ensuite, on note une méconnaissance réciproque, un cloisonnement recherche/industrie. Deuxièmement, on ne peut que constater la faiblesse des liens entre entreprises locales. Les entreprises régionales sont atomisées. Elles sont soit en forte concurrence, soit elles s'ignorent purement et simplement. La trop grande diversité des problématiques et des situations individuelles empêche de trouver des 
problématiques communes. Les expériences de coopération sont bien souvent limitées à la mutualisation de moyens (investissement en commun sur des machines). Le seul nœud de communication, le seul point d'appui aux coopérations locales bien identifié est le salon Micronora. C'est d'ailleurs souvent la seule action collective consommée : en dehors de Micronora, les entreprises déclarent ne pas avoir de projets, ni de besoins particuliers en coopération ou en actions collectives, en dehors de l'aspect commercial (ex service commercial commun, une prospection dans les pays auquel la petite structure n'a pas accès).

\section{B. L'émergence d'un système microtechnique local depuis 2001}

Nous venons de mettre en évidence le rôle du système éducatif et de recherche dans l'émergence de l'industrie microtechnique. Jusqu'en 2001, néanmoins, on ne pouvait que constater le cloisonnement entre acteurs, nous faisant douter de l'existence d'un système microtechnique au niveau local. À partir de cette date, une succession de pressions institutionnelles et/ou d'initiatives locales marquent l'évolution dans les relations au sein du système. Le local autour des microtechniques tend à se développer. Le rôle d'institutions comme le Conseil économique et social régional (CESR), le rôle d'industriels (UIMM et comité des microtechniques) et les effets des politiques d'aménagement du territoire (SPL et pôles de compétitivité) sont pour beaucoup dans l'émergence de proximités et de relations au sein du système microtechniques. L'émergence de relations locales n'en reste pas moins qu'au stade du balbutiement et demande maturation et pérennisation.

\section{L'émergence de relations inter-entreprises}

21 Une autosaisine du CESR sur « l'avenir des microtechniques en Franche-Comté » (CESR, 2001), largement critiquée pour son caractère pessimiste, a néanmoins agi comme catalyseur de la problématisation des microtechniques comme objet de développement local. Le rapport souhaitait dresser un état des lieux objectif de la situation des microtechniques. Tentant de cerner la notion de microtechniques, il proposait de considérer comme microtechnique tout composant dont la taille était de l'ordre du mm3 ou tout composant dont l'énergie requise pour assurer ses fonctions était de l'ordre du microwatt. Pour lui, un indice fort était la présence ou non d'une salle blanche pour la fabrication de l'objet. Il excluait donc explicitement l'horlogerie et la micromécanique du champ des microtechniques, soit l'essentiel du tissu régional. Cette définition fut mal accueillie, jugée trop restrictive et trop scientifique. Le rapport identifie ensuite sur la base de cette définition et de répertoires existants les entreprises dont l'activité serait liée aux microtechniques et aboutit à la conclusion que les microtechniques se réduiraient à peu de choses... 30 entreprises, pour à peine plus de 1000 emplois. De nombreux acteurs se sont insurgés des résultats.

Ce rapport, bien que discrédité à l'époque, eut des effets marquants dans l'évolution de la problématique autour des microtechniques.

Premièrement, il permit d'engager une réflexion collective et publique sur l'avenir des microtechniques en Franche-Comté qui aboutit à la conclusion de la nécessité de développer des démarches collectives. Entre 2002 et 2003, plusieurs réunions, ouvertes à tous les acteurs de la vie économique, permirent de débattre des résultats du rapport de l'autosaisine et d'avancer la réflexion régionale sur le sujet. Deuxièmement, il eut 
l'avantage de conduire pour la première fois à une mobilisation de certaines entreprises, soit offusquées de ne pas apparaître comme entreprises microtechniques dans le rapport, soit au contraire flattées de l'être. Enfin, le CESR a souhaité créer un groupe de réflexion sur l'avenir des microtechniques en Franche- Comté, composé uniquement d'industriels et organisa des réunions constructives entre 2002 et 2003. Le CESR a permis d'instaurer la phase préalable nécessaire de mise en place progressive de relations de confiance dans le cadre de rencontres informelles : rencontres interentreprises, visites... ont permis aux industriels de mieux se connaître, d'échanger des informations, d'envisager ce qu'il était possible de faire ensemble, de mettre le doigt sur des complémentarités possibles, bref de découvrir qu'il existait localement des compétences que l'on était donc pas forcément obligé d'aller chercher ailleurs. Les industriels ayant ainsi abouti à identifier des problématiques communes et définir des d'objectifs communs, le CESR a donné une nouvelle impulsion en permettant la création d'un club microtechnique. Les relations se sont donc formalisées et des règles d'information réciproque se sont instaurées (Club privé avec ses propres conventions, ses propres règles d'information réciproque et de transparence pour ne pas piller les autres, pour organiser la facturation...). Un climat de confiance s'est installé. Progressivement, le club a engagé des projets collectifs. Baptisé Comité des microtechniques ${ }^{6}$, il a été accueilli au sein de l'UIMM. Par ce biais, l'UIMM pouvait enfin commencer à prétendre représenter, au plan régional, les professions de ce secteur d'activité. La DRIRE ou le Conseil régional avaient de leur côté désormais des interlocuteurs autres que des directeurs de laboratoire ou des universitaires et ainsi orienter les décisions en fonction des besoins des industriels. Le comité s'est doté d'une Charte, qui n'exclut personne mais règle des points de professionnalisme et de confidentialité. Il s'est fixé un programme, un plan d'actions, une réflexion stratégique. Il a orienté sa démarche vers la préparation d'un Contrat professionnel de Progrès en faveur des métiers des microtechniques ${ }^{7}$. De premières actions de coopération autour d'actions simples à mettre en œuvre ont pu ainsi voir le jour. La première action collective en date fut un stand commun au salon Micronora 2004.

Même si les impulsions institutionnelles ont été nécessaires pour instaurer un rapprochement d'industriels autour de la problématique des microtechniques, elles n'auraient cependant pas été suffisantes sans l'action de ce petit groupe de PME volontaires et actives. Les impulsions institutionnelles, pour qu'elles prennent effet, doivent pouvoir s'appuyer sur des énergies locales. Les 8 industriels fondateurs du comité microtechnique ont pu constituer un noyau dur en raison d'une proximité d'âge, de valeurs communes, de problématiques partagées, d'une même vision de l'avenir... un ciment nécessaire à la constitution d'un réseau de compétences. Aujourd'hui, le comité microtechnique compte une trentaine de membres. Même s'il n'atteint pas la masse critique suffisante et n'est pas encore véritablement légitime en terme de représentativité du secteur microtechnique, il peut constituer un support à l'émergence de relations interentreprises.

\section{La structuration de la recherche et du transfert}

Parallèlement à la volonté de structuration constatée du côté des industriels, la recherche et le transfert tendent eux aussi à s'organiser.

Du point de vue de la recherche publique, il existe en Franche-Comté depuis plusieurs décennies une concentration de laboratoires publics dans le domaine des Sciences de 
l'Ingénieur, dont la plupart sont associés au CNRS et reconnus internationalement. La structuration de ces laboratoires sur le domaine des microtechniques a débuté dès le début des années 1990. À la suite de l'émergence des technologies des «Microsystèmes » (ou "MEMS " ${ }^{8}$ aux États-Unis ou encore "Micromachines » au Japon), on a constaté l'existence dans plusieurs laboratoires CNRS et associés d'une composante microtechnique plus ou moins développée. Pour des raisons de taille critique des laboratoires, d'un passé horloger prestigieux et surtout par souci d'efficacité, il fut alors décidé de tenter un rapprochement thématique des laboratoires les plus impliqués en Franche-Comté dans le domaine des Microtechniques. C'est ainsi que fut créé en 1990 l'Institut des Microtechniques de Franche-Comté (IMFC). En 1996, il fédérait 7 laboratoires en sciences de l'ingénieur'. L'IMFC a fédéré ce secteur pendant 10 ans, en permettant la constitution d'une plateforme technologique commune (la plate-forme technologique Mimento) et en suscitant des programmes de développement spécifiques. En 2004, la centrale de technologie franc-comtoise Mimento a été labellisée par le ministère de la recherche comme un équipement d'envergure nationale. Elle fait maintenant partie des 6 centrales de technologie du réseau RTB (réseau des technologies de base) en micro et nano technologies.

Récemment, cinq des laboratoires fédérés par l'IMFC ont effectué un pas supplémentaire en fusionnant purement et simplement pour créer au 1er janvier 2004 l'Institut Femto-ST (Franche-Comté Électronique, Mécanique, Thermique et Optique Sciences et Technologies), qui remplace donc l'IMFC à présent. Cette nouvelle organisation de la recherche publique en Franche-Comté dans les domaines des "Sciences et Technologies de l'Information et de la Communication» (STIC) et des "Sciences Pour l'ingénieur» (SPI) est sensée mieux animer le secteur des Microtechniques en Franche-Comté. Les activités de recherche de Femto-ST sont à présent regroupées en quatre axes: microfabrication micro et nanosystèmes ; ingénierie biomédicale ; temps-fréquence et télécommunications ; énergie et environnement. Mais les recherches de Femto-ST portent sur des problématiques hitech concernant peu d'entreprises régionales et, on ne peut que le constater, le champ de recherche est beaucoup plus large que les microtechniques. Les problématiques micromécaniques sont plutôt portées par le LAB - Laboratoire d'Automatique de Besançon -, écarté de Femto-ST alors qu'il était dans l'IMFC, ce qui est révélateur L'institut de recherche Femto-ST, en association avec le Centre de Transfert des Micro et Nanotechnologies (CTMN), fait désormais partie depuis mars 2006 des premières structures ayant obtenu le label Carnot sous l'appellation « Femto INNOVATION ». Ce label est décerné par le Ministère de l'Enseignement Supérieur et de la Recherche à des organismes de recherche nationaux qui, tout en continuant à mener une activité de recherche fondamentale de haut niveau, ont mis en place une organisation interne favorisant le développement de travaux partenariaux avec le monde socioéconomique.

Du point de vue du transfert des technologies, la création du Centre de Transfert des Microtechniques (CTM) en 1999 était faite pour remédier à l'absence véritable de structure d'interface entre chercheurs et industriels. Constitué sous la forme d'un groupement d'intérêt public, il associait le CETEHOR, l'Université de Franche-Comté, le CNRS et six entreprises (Cheval Frères, Framatome, France Ébauches Microtechniques, Hydromécanique et Frottement, Isa France et PZT International)

En fait, peu d'industriels locaux étaient concernés par les activités du CTM. Peu à peu, des problèmes de gestion sont survenus et ont mis le CTM en péril. 
31 Alors que le CTM était en crise, les demandes des industriels ont été analysées. On s'est très vite rendu compte qu'ils n'étaient pas demandeurs d'un centre de transfert pour la science, mais plutôt d'un centre technique. Autant le Cetehor était utile à l'horlogerie, autant il n'existait pas d'équivalent pour les microtechniques. Le centre technique au profit de l'horlogerie n'était en effet plus adapté aux microtechniques puisqu'il est désormais orienté vers l'or.

Côté transfert de la recherche vers l'industrie, le CTM a bien transféré, mais pas vers des entreprises du territoire ; elle transférait peu localement car il y avait peu de demandeurs (à part Statice et Cheval).

Un nouveau projet aboutit avec le successeur du CTM : l'association de loi 1901 Centre de Transfert en Micro et Nanotechnologies (CTMN) est crée en 2004 avec pour double objectif d'être un centre de transfert à l'extérieur et un centre technique localement (prêt d'ingénieurs à un prix correct, atelier pilote dans la maison des microtechniques prêté aux entreprises - microinjection, laser Femto...).

34 Le CTMN regroupe des organismes de recherche (Université de Franche-Comté, Université Technologique de Belfort-Montbéliard, ENSMM), des représentants de l'industrie régionale (UIMM, Comité microtechnique, syndicat des composants horlogers et microtechniques...), des industriels régionaux (Cheval frères, Technotime...) et des industriels hors région (Radiall).

Une nouvelle initiative marque une évolution dans l'organisation de la recherche et du transfert dans les microtechniques en Franche-Comté. Mis en place au début de l'année 2007 dans le cadre du contrat de plan État-région, l'institut Pierre Vernier favorise les transferts de technologie dans toute la région Franche- Comté. À l'image de l'institut Femto-ST qui a regroupé en 2004 cinq laboratoires régionaux de recherche, l'institut Pierre Vernier fédère les trois principaux établissements d'enseignement supérieur de la région - l'Université de Franche-Comté, l'Université de technologie de BelfortMontbéliard et l'ENSMM de Besançon - ainsi que les trois grands centres de transfert technologique : le Centre de transfert des micro et nanotechnologies, l'Institut de productique et le pôle régional de conception et d'innovation.

L'industrie microtechnique s'est donc dotée depuis 2001 de cadres d'action bien identifiés en faveur des microtechniques, dans l'industrie comme dans la recherche ou le transfert. Le Pôle de compétitivité, le comité microtechnique, le CTMN, Femto-ST, l'institut Pierre Vernier, Micronora etc. sont autant de supports visibles pour le développement de relations entre acteurs de l'innovation.

\section{Conclusion}

37 Le cas de la reconversion des espaces horlogers français, véritables districts industriels en crise, et le développement territorial autour des microtechniques fournit trois éclairages précieux. Premièrement, il montre le rôle clé du système éducatif, un rôle souvent sousestimé ou peu étudié dans la constitution d'organisations territoriales comme les SPL. Deuxièmement, il confirme que la réussite d'un SPL ne se décrète pas, chaque acteur étant soumis à des contraintes propres qui ne facilitent pas la coopération territoriale. Troisièmement, les conditions de développement du territoire sont déterminantes dans l'évolution d'un système productif. Dans notre cas, la nature des entreprises horlogères étant empreinte de tradition mécanique, le tissu industriel 
local est essentiellement micromécanique, exclut la microélectronique et ne peut trouver d'interlocuteurs dans un système éducatif et de recherche proposant des solutions microtechniques à technologie poussée. L'éclatement du système horloger a donné naissance à un système beaucoup plus complexe, constitué d'une multitude d'entreprises surtout en position de sous-traitance face à des donneurs d'ordre nombreux et d'horizons divers. Les industries locales ne trouvent donc plus un débouché dans un produit commun et dans une filière unique comme c'était le cas pour l'horlogerie. Elles sont désormais en position de sous-traitance, sur des filières multiples dans lesquelles elles ont dû s'insérer. Entreprises innovantes et efficaces, elles sont désormais plus liées à leurs donneurs d'ordre qu'à leur environnement de proximité. Il est dès lors difficile de jeter les bases d'un système productif localement identifié, dans un contexte où les activités finales sont trop diversifiées, les entreprises trop petites et en situation de trop forte concurrence pour les unifier autour d'un projet commun, avec des institutions professionnelles représentatives.

Il a fallu une série de crises, d'évolutions nationales ou régionales, l'initiative de quelques pionniers pour que, à partir de 2001, les microtechniques commencent à devenir un objet intéressant de développement territorial. Les relations interentreprises et entre entreprises, éducation et recherche commencent à émerger et ont désormais des structures dédiées (pôle de compétitivité, comité microtechnique...) ; elles doivent néanmoins se développer et être pérennisées pour que l'industrie microtechnique puisse se positionner dans la compétition nationale, européenne voire mondiale.

\section{BIBLIOGRAPHIE}

CAREL S. (2005). - La politique française de développement de réseaux d'entreprises localisés. Des SPL aux pôles de compétitivité, quels enjeux pour les territoires ? Dans Actes des septièmes rencontres de Théoquant. Besançon : ThéMA, Université de Franche-Comté.

CAREL S. (2003). - Rapports complexes entre Industrie et Territoire : le secret microtechnique franc-comtois. Dans Actes du XXXIXe colloque de l'ASRDLF. Lyon.

CAREL S. (2003). - Système productif complexe et pluriterritorial. Dans Actes des sixièmes rencontres de Théoquant. Besançon : ThéMA, Université de Franche-Comté.

CAREL S., LARCENEUX A. (2004). - De l'horlogerie aux microtechniques. La trajectoire d'un SPL franc-comtois. Dans J.-C. Daumas, Les systèmes productifs dans l'Arc jurassien. Acteurs, pratiques et territoires (XIXe-XXe siècles). Besançon : Presses Universitaires de Franche-Comté.

CAREL S., LARCENEUX A. (2004). - La difficile reconversion de l'horlogerie franc-comtoise vers les microtechniques. Dans Formation-Emploi (Vol. $n^{\circ} 87$ ). Paris : La Documentation française.

CHEREF S. (1996). - Les microtechniques : une spécialisation de Besançon. Dans S. Cheref, J.-C. Chevailler, A. Larceneux, Le développement d'une capitale régionale : Besançon, citadelle assiégée ou métropole en devenir ? Besançon : Irades. 
CHEREF S., CUISINIER P., KABANTCHENKO E., LARCENEUX A., TERNANT E. - La rénovation de la filière microtechnique (Vol. CPC documents 98/3). 1998 : Irades, Ministère de l'Education nationale. Conseil économique et social de Franche-Comté. (2001). - Quel avenir pour les microtechniques en Franche-Comté? Autosaisine, mai 2001. Besançon.

MAILLAT D., NÉMETI F., PFISTER M., SIVIERO A. (1992). - Les microtechniques et les services associés : quels espaces de production et de coopération pour la Suisse? Neuchâtel.

MAILLAT D., NÉMETI F., PFISTER M., SIVIERO A. (1993). - L'industrie microtechnique en Suisse. Neuchâtel : IRER, EDES.

TERNANT E. (2003). - La dynamique longue d'un système productif localisé : l'industrie de la montre en Franche-Comté. Thèse de doctorat. Université Pierre Mendès France, Grenoble.

TERNANT E. (2004). - L'affaiblissement du SPL horloger franc-comtois : mythes et réalités historiques. Dans J.-C. Daumas, Les systèmes productifs dans l'Arc jurassien. Acteurs, pratiques et territoires (XIXe-XXe siècles). Besançon : Presses Universitaires de Franche- Comté.

TERNANT E., ODOUZE A.-M. (1995). - De l'horlogerie aux microtechniques 1965-1975. Actes du colloque organisé par le Cétéhor et le Musée du Temps. Besançon.

UIMM et Comité des microtechniques de Franche-Comté. (2005). - Pôle de compétitivité des microtechniques. Un enjeu pour la région Franche-Comté, un enjeu pour la France et l'Europe. Dossier de candidature remis au Gouvernement, 28 février 2005. Besançon.

\section{NOTES}

1. Deux objectifs quantitatifs ont été fixés: Une intensité de Recherche-Développement (dépenses de RD en \% du PIB) de $3 \%$ pour l'ensemble de l'Union européenne, et - 2/3 des dépenses de Recherche-Développement financées par le secteur des entreprises

2. Six pôles «mondiaux " qui disposent d'ores et déjà d'un rayonnement international et neuf pôles « à vocation mondiale » qui ont un potentiel international à développer.

3. La décision du CIADT de décembre 1997 de «soutenir les initiatives tendant à consolider ou faire émerger des systèmes productifs locaux» a conduit au lancement de deux appels à projets en novembre 1998 et novembre 1999. Sur 202 réponses à l'appel, la DATAR en a sélectionné 96.94 ont été effectivement engagés au cours des années 1999 et 2000, dont le SPL bisontin microtechnique.

4. Salon européen des microtechniques, organisé tous les deux ans à Besançon.

5. Entreprise horlogère bisontine célèbre pour tous les problèmes sociaux et syndicaux qui ont défrayé la chronique pendant des années, la prise de possession de l'usine par le personnel, la vente des stocks dite "vente sauvage ». Les activités de la marque ont été reprises en 1990 par Jean-Claude Sensemat qui fonda la société LIP France.

6. Le comité a été fondé par les 8 entreprises suivantes : AR Électronique, Cryla, Digital Surf, Photline Technologies, Imasonic, Morel Technic, Nanobiogène, Statice.

7. Il existe déjà un Contrat professionnel de Progrès au bénéfice des métiers du découpageemboutissage en Franche-Comté, établi le 20 janvier 2003.

8. Micro-Electro-Mechanical-Systems

9. Le LPMO (Laboratoire de Physique et Métrologie des Oscillateurs), Le LOPMD (Laboratoire d'Optique de P.M. Duffieux), le LMARC (Laboratoire de Mécanique Appliquée R. Chaléat), le LAB (Laboratoire d'Automatique de Besançon), le LCEP (Laboratoire de Chronométrie, Électronique et Piézoélectricité), l'IGE (Institut de Génie Énergétique) situé à Belfort, le LMIT (Laboratoire de Métrologie des Interfaces Techniques) situé à Montbéliard. 


\section{RÉSUMÉS}

Cet article fait le point sur près de quatre décennies d'existence et d'évolution de l'industrie microtechnique en Franche-Comté. Le cas de la reconversion des espaces horlogers français, véritables districts industriels en crise et le développement territorial autour des microtechniques fournissent des éclairages précieux sur les difficultés de structuration territoriale d'une industrie malgré la pression de politiques volontaristes.

This article reviews nearly four decades of change in the microtechnical industry of FrancheComté. The case of the reconversion of French clockmakers, industrial districts in real crisis and territorial development around microtechnical industries, sheds invaluable light on the difficulties of restructuring an industry, despite the pressure of voluntary politics.

Dieser Artikel gibt einen Überblick über die nahezu vier Jahrzehnte dauernde Entwicklung der Mikrotechnik-Industrie in der Franche-Comté. Der Fall der Umgestaltung der französischen Uhrenrindustrie-Region, einem Industriedistrikt in der Krise, und die territoriale Entwicklung rund um die Mikrotechnik erklären, welchen Schwierigkeiten regionale Strukturationsprozesse trotz gezielter Wirtschaftspolitik ausgesetzt sind.

\section{INDEX}

Mots-clés : Franche-Comté, microtechniques, pôles de compétitivité, système productif local Schlüsselwörter : Franche-Comté, Industriedistrikt, Innovationsund Technologiezentren, lokales produktives System, Mikrotechnik

Keywords : centres of competitivness, Franche-Comté, local production system, microtechnical

\section{AUTEUR}

\section{SOPHIE CAREL}

Université de Franche-Comté - Laboratoire ThéMA UMR6049 - Besançon - sophie.carel@univfcomte.fr 